\title{
Berufliche Aus- und Weiterbildung in Unternehmen
}

Die ökonomische, technologische und demografische Entwicklung wird die Aus- und Weiterbildung in den Unternehmen in Deutschland künftig vor große Herausforderungen stellen. Das abnehmende Beschäftigungsreservoir wird voraussichtlich dazu führen, dass Unternehmen auch weniger qualifizierte Arbeitskräfte integrieren und entsprechend qualifizieren müssen. Der Beitrag beschreibt das Aus- und Weiterbildungsangebot in Unternehmen. Dabei werden insbesondere die Organisation der beruflichen Bildung und die innerbetriebliche Zusammenarbeit bei der Aus- und Weiterbildung als eine wesentliche Voraussetzung für eine Verbesserung der Bildungsbeteiligung der Unternehmen in quantitativer und qualitativer Hinsicht betrachtet.

DICK MORAAL, GUDRUN SCHÖNFELD

\section{Einleitung}

Aus- und Weiterbildung in Unternehmen sind ein wichtiges Instrument zur Sicherung des Fachkräftebedarfs der Wirtschaft. Sie ermöglichen den Jugendlichen die Integration in den Arbeitsmarkt und den Arbeitskräften den Erhalt ihrer Beschäftigungsfähigkeit. Den Unternehmen sichern sie ihre Innovations- und Wettbewerbsfähigkeit gerade auch mit Blick auf den demografischen Wandel und die sich rasch ändernden technischen Entwicklungen. Die von der Politik propagierte Forderung nach einem kontinuierlichen lebenslangen Lernen stimmt aber nicht unbedingt mit den Realitäten in den Unternehmen überein. Bei der Ausbildung ist hier zum Beispiel an die immer noch existierende strukturelle Knappheit bei den Ausbildungsplätzen zu denken; bei der Weiterbildung an oft nur auf Anpassungsmaßnahmen ausgerichtete betriebliche Weiterbildungsangebote, die nicht die Arbeitsmarktmobilität der Beschäftigten berücksichtigen.

Vor diesem Hintergrund beschreibt der Artikel anhand der dritten europäischen Erhebung zur betrieblichen Weiterbildung (CVTS3; Continuing Vocational Training Survey) das Aus- und Weiterbildungsangebot der Unternehmen in Deutschland. Als mögliche Ursache für die Defizite werden dabei insbesondere Schwächen in der Organisation der Weiterbildung, beim Angebot an differenzierten Lernformen und dem Zusammenspiel zwischen der betrieblichen Aus- und Weiterbildung angesprochen.

\section{Theoretischer Hintergrund}

\subsection{Betriebliche Erstausbildung: nachlassende Aktivität der Unternehmen}

Aktuell genießt die duale Ausbildung in Deutschland international große Wertschätzung und wird vermehrt als Vorbild für Reformen in anderen Ländern genutzt. Insbesondere die im europäischen Vergleich niedrige Jugendarbeitslosigkeit, der hohe Qualifikationsstand der Beschäftigten und der relativ gute Übergang in den Arbeitsmarkt nach der Ausbildung werden als wichtige Vorteile identifiziert (BMBF 2012). Dies sollte aber nicht darüber hinwegtäuschen, dass auch in Deutschland nicht alles Gold ist, was glänzt.

Die Bereitstellung einer ausreichenden Zahl von Ausbildungsplätzen ist die wichtigste Voraussetzung für den Erfolg des dualen Systems. Dies gelang in den letzten Jahren jedoch nicht in ausreichendem Maße. Zwar ändert sich jetzt langsam die Situation zugunsten der Jugendlichen, da einerseits die Zahl der Schulabgänger abnimmt und andererseits sich der Trend zur Hochschulausbildung verstärkt. Dennoch liegt die Angebots-Nachfrage-Relation 2011 nur bei $92,7 \%$ und es bestehen starke regionale und be-

Die Angebots-Nachfrage-Relation gibt wieder, wie viele Ausbildungsplatzangebote rechnerisch auf 100 Ausbildungsplatznachfrager entfallen (siehe Ulrich et al. 2011, S. 14). 
rufliche Ungleichgewichte auf dem Ausbildungsmarkt (Ulrich et al. 2011). Von einer wirklichen Wahl kann also weiterhin nicht gesprochen werden.

Bedenklich ist auch die Entwicklung bei der Zahl der ausbildenden Betriebe. Bildeten in den 1980er Jahren (alte Bundesländer) noch mehr als ein Drittel aller Betriebe aus, reduzierte sich seitdem die Zahl stetig. Im Jahr 2010 bildeten nur noch 22,5\% aller Betriebe aus, ein nochmals deutlicher Rückgang zu den 24,1 \% des Jahres 2005 (BIBB 2012). Insbesondere kleinere Unternehmen zogen sich zurück. Eine Langzeituntersuchung zur Nachhaltigkeit betrieblicher Ausbildungsbeteiligung ergab, dass sich von den zwischen 1999 und 2008 registrierten Betrieben 72,9 \% grundsätzlich nicht an der Ausbildung beteiligten und nur 3,4 \% im gesamten Zeitraum Jugendliche in Ausbildung hatten. Dabei fällt das durchschnittliche Ausbildungsstellenangebot im Betrieb desto höher aus, je länger die Beteiligung an Ausbildung dauert (Troltsch 2010).

In Verbindung mit dem strukturellen Wandel von der Industrie- zur Dienstleistungs- und Wissensgesellschaft führen diese Entwicklungen dazu, dass seit Langem eine große Zahl von Jugendlichen außen vor bleibt und in das Übergangssystem $^{2}$ verwiesen wird. Die Zugangszahlen sind zwar rückläufig, dennoch waren 2011 rund 300.000 junge Menschen betroffen (BIBB 2012). Besonders kritisch ist hierbei, dass die Bildungsgänge nicht zu einem qualifizierten Berufsabschluss führen und nur selten auf später folgende Ausbildungsmaßnahmen angerechnet werden. Sie tragen so nicht zu einer Durchlässigkeit des Bildungssystems bei. ${ }^{3}$

\subsection{Segmentierung im deutschen Bildungssystem}

Bildungsforscher weisen immer wieder darauf hin, dass das berufliche Aus- und Weiterbildungssystem in Deutschland weitgehend segmentiert ist. Baethge (2007, S. 24) charakterisiert diese Segmentierung folgendermaßen: Bildungssysteme lassen sich „danach unterscheiden, wie sehr sie durch Grenzen segmentiert und wie durchlässig oder undurchlässig die Grenzen sind, was davon abhängig ist, wie stark die einzelnen Bildungsbereiche unterschiedlichen institutionellen Ordnungen folgen. Das deutsche Bildungssystem ist mit der Dreigliedrigkeit seines allgemeinbildenden Schulwesens und der strikten institutionellen Trennung von Allgemeinbildung und Berufsbildung sowie Hochschule und Weiterbildung wie kaum ein anderes Bildungssystem in der Welt segmentiert."

Das duale Ausbildungssystem war allerdings lange Zeit kollektivistisch geprägt und wurde im Zusammenwirken von Staat und Sozialpartnern gesteuert. Vorherrschend waren ein hohes Maß an Kooperationsbereitschaft und der Wille zur Einigung (Konsensprinzip). Bedingt durch die Dezentralisierung im System der industriellen Beziehungen in den letzten beiden Jahrzehnten hat jedoch ein allmähli- cher Wandel von einem kollektivistischen zu einem stärker segmentalistischen Modell stattgefunden (vgl. zum Beispiel Thelen/Busemeyer 2012; Trampusch 2010). Dabei gelingt es einzelnen Gruppen, insbesondere größeren Industrieunternehmen und ihren Verbänden, eher ihre Partikularinteressen auf Kosten gemeinsamer Interessen aller Unternehmen durchzusetzen. Beispielhaft seien hier Forderungen nach einer größeren Flexibilisierung, Dezentralisierung und Ausrichtung der Ausbildung auf betriebsspezifische Bedürfnisse genannt. Kennzeichen segmentierter Systeme ist die Ausbildung vor allem für den internen Arbeitsmarkt, in kollektivistischen Systemen beteiligt sich eine größere Zahl von Unternehmen, einschließlich kleinerer, an der Ausbildung (Thelen/Busemeyer 2012, S. 71).

Bei der Weiterbildung wird zwischen den drei Teilbereichen der betrieblichen Weiterbildung, der individuellen beruflichen Weiterbildung und der Weiterbildung für Arbeitslose und von Arbeitslosigkeit bedrohte Arbeitnehmer unterschieden (Sauter 2003). Die drei Bereiche sind institutionell stark segmentiert. Die Verantwortlichkeiten liegen bei den jeweiligen Akteuren selbst - beim Staat für die Weiterbildung der Arbeitslosen und von Arbeitslosigkeit bedrohten Arbeitnehmer, bei den Unternehmen für die betriebliche Weiterbildung und bei den Individuen für ihre eigene berufliche Weiterbildung. Das Prinzip der Subsidiarität (Verantwortung im eigenen Bereich) ist sehr stark entwickelt. Diese institutionelle Segmentierung der unterschiedlichen Bereiche der beruflichen Weiterbildung setzt damit die strukturellen Rahmenbedingungen für deren Implementierung und begrenzt die Möglichkeiten einer effizienten Zusammenarbeit zwischen den verschiedenen Akteuren in der beruflichen Weiterbildung. ${ }^{\oplus}$

(2) Dies sind "(Aus-)Bildungsangebote, die unterhalb einer qualifizierten Berufsausbildung liegen beziehungsweise zu keinem anerkannten Ausbildungsabschluss führen, sondern auf eine Verbesserung der individuellen Kompetenzen von Jugendlichen zur Aufnahme einer Ausbildung oder Beschäftigung zielen und zum Teil das Nachholen eines allgemein bildenden Schulabschlusses ermöglichen" (Konsortium Bildungsberichterstattung 2006, S. 79).

(3) Insbesondere Jugendliche, die eigentlich die erforderlichen Voraussetzungen für eine Berufsausbildung besitzen, stecken so häufig in unnötigen und ineffektiven Warteschleifen. Nicht ausbildungsreife Jugendliche oder solche, die einen höheren Schulabschluss erreichen wollen, profitieren eher von den Maßnahmen (Beicht 2010, S. 96).

(4) In anderen Ländern wie Dänemark oder die Niederlande sind die Akteure im Bereich der beruflichen Weiterbildung (Staat, Unternehmen, Gewerkschaften, Individuen und Bildungsträger) in ein komplexes System kombinierter Verantwortlichkeiten für die berufliche Weiterbildung eingebunden, was die Zusammenarbeit beim Aufbau eines effektiven und nachhaltigen Konzepts des lebenslangen Lernens vereinfacht. 


\subsection{Betriebliche Weiterbildung: im internatio- nalen Vergleich nur mittelmäßig}

Gilt das deutsche duale Ausbildungssystem an sich wegen seiner Praxisnähe und der niedrigen Jugendarbeitslosigkeit im europäischen Vergleich als vorbildlich - insbesondere für die Jugendlichen, denen der direkte Einstieg unmittelbar nach Schulabschluss gelingt, - trifft dies auf die Weiterbildung, sofern man sich auf die betriebliche Weiterbildung beschränkt und die individuelle berufliche Weiterbildung nicht berücksichtigt, nicht unbedingt zu. So findet sich Deutschland nach den Ergebnissen der dritten europäischen Erhebung zur betrieblichen Weiterbildung (CVTS3; Continuing Vocational Training Survey) ${ }^{\complement}$ beim Weiterbildungsengagement nur im europäischen Mittelfeld wieder (Behringer/Schönfeld 2010): Der Anteil der weiterbildenden Unternehmen und die Weiterbildungsintensität gemessen in Kursstunden je 1000 Arbeitsstunden war zwar im Jahr 2005 leicht überdurchschnittlich, die Teilnahmequote an Kursen und auch die Ausgaben der Unternehmen als Anteil an den Gesamtarbeitskosten aber unterdurchschnittlich. Eine Spitzenstellung nahmen die Unternehmen hingegen bei den arbeitsplatznahen Lernformen wie Einarbeitung und Unterweisung durch Vorgesetzte oder Kollegen, Lern- und Qualitätszirkel oder dem selbstgesteuerten Lernen ein (Behringer/Käpplinger 2011).

Nach dem Betriebspanel des Instituts für Arbeitsmarktund Berufsforschung (IAB) ist in Deutschland nicht einmal jeder zweite Betrieb weiterbildungsaktiv. Die Weiterbildungsaktivität hat dabei in den letzten Jahren leicht zugenommen (von $36 \% 2001$ auf $45 \%$ 2009) und ist positiv mit der Betriebsgröße korreliert (Stegmaier 2010). Erheblich höhere Zahlen weist das Institut der deutschen Wirtschaft (IW) in seiner Weiterbildungserhebung für das Jahr 2010 aus - $83 \%$ der Unternehmen boten Weiterbildung an, das Stundenvolumen bei Kursen stieg um mehr als ein Drittel im Vergleich zu 2007. Die Kosten blieben jedoch konstant (Seyda/Werner 2012).

Als mögliche Ursache für die niedrige Weiterbildungsbeteiligung wird häufig auf die geringere Notwendigkeit angesichts des soliden Fundaments durch die Erstausbildung verwiesen. Gerade vor dem Hintergrund des demografischen Wandels und des befürchteten Fachkräftemangels wird aber zunehmend sowohl auf nationaler als auch auf europäischer Ebene auf die große Bedeutung der Weiterbildung hingewiesen, um die Qualifikationen aktuell zu erhalten beziehungsweise an neue Anforderungen anzupassen.

\section{Datenbasis}

Wie sieht es nun aber konkret mit der beruflichen Bildung in den Unternehmen in Deutschland aus? Im Mittelpunkt der folgenden Auswertungen stehen das Aus- und Weiterbil- dungsangebot der Unternehmen, insbesondere mit Blick auf die Nutzung verschiedener Lernformen, die Organisation der Weiterbildung und die gemeinsame Nutzung von Ressourcen sowohl für die Aus- als auch für die Weiterbildung. Es werden die deutschen CVTS3-Mikrodaten genutzt. Dieser Datensatz eignet sich für dieses Thema besonders, da dort neben Fragen zur betrieblichen Weiterbildung ${ }^{\odot}$ erstmals auch einige Informationen über die betriebliche Erstausbildung (Teilnehmende, Kosten) erhoben wurden. Befragt wurden Unternehmen mit zehn und mehr Beschäftigten aus den Wirtschaftsbereichen Verarbeitendes Gewerbe, Handel, Kredit- und Versicherungsgewerbe, Baugewerbe, Energie- und Wasserversorgung, Bergbau und Gewinnung von Steinen und Erden, Verkehr und Nachrichtenübermittlung, Gastgewerbe und unternehmensnahe sowie sonstige öffentliche und persönliche Dienstleistungen. ${ }^{\circledR}$ Insgesamt beteiligten sich 2.857 Unternehmen an der schriftlichen Befragung.

Ergänzend werden die Daten der CVTS3-Zusatzerhebung des Bundesinstituts für Berufsbildung (BIBB) herangezogen. 2008 wurden 302 weiterbildende Unternehmen, die auch an CVTS3 teilgenommen haben, telefonisch zu qualitativen Aspekten der betrieblichen Weiterbildung befragt. Die Verzahnung von Aus- und Weiterbildung in Unternehmen bildete einen Schwerpunkt der Befragung (Moraal et al. 2009).

\section{Ergebnisse}

\subsection{Bildungsaktivitäten der Unternehmen}

Grundsätzlich muss sich kein Unternehmen an betrieblichen Qualifizierungsmaßnahmen beteiligen, da es theoretisch Arbeitskräfte mit den benötigten Qualifikationen auch über den Arbeitsmarkt anwerben könnte. Auch gibt es

(5) Die Erhebungen liefern in bisher vier Befragungswellen für die Jahre 1993, 1999, 2005 und 2010 (CVTS1-4) grundlegende Informationen über das Angebot, die Formen, Inhalte, Teilnehmende, den Umfang und die Kosten der betrieblichen Weiterbildung in Europa (vgl. Cedefop 2010). Ergebnisse liegen für die ersten drei Erhebungen vor, deutsche CVTS4-Ergebnisse sollen im Herbst 2012 vom Statistischen Bundesamt veröffentlicht werden, europäische erst 2013. Die nachfolgend dargestellten Strukturen für Deutschland sind im Wesentlichen seit der ersten Erhebung 1993 gleichgeblieben.

(6) Definition der betrieblichen Weiterbildung: Vorausgeplantes, organisiertes Lernen, das vollständig oder teilweise von den Unternehmen finanziert wird.

(7) Die öffentliche Verwaltung, Verteidigung, Sozialversicherung, Erziehung und Unterricht und das Gesundheits-, Veterinär- und Sozialwesen sind die wesentlichen Wirtschaftsbereiche, die fehlen. 


\section{Bildungsaktivität der Unternehmen/Betriebe}

in Prozent aller Unternehmen/Betriebe

\begin{tabular}{|c|c|c|c|c|c|c|c|c|}
\hline & \multicolumn{2}{|c|}{$\begin{array}{l}\text { Aus- und weiterbildende } \\
\text { Unternehmen / Betriebe }\end{array}$} & \multicolumn{2}{|c|}{$\begin{array}{l}\text { Nur weiterbildende } \\
\text { Unternehmen / Betriebe }\end{array}$} & \multicolumn{2}{|c|}{$\begin{array}{c}\text { Nur ausbildende } \\
\text { Unternehmen / Betriebe }\end{array}$} & \multicolumn{2}{|c|}{ Keine Bildungsaktivität } \\
\hline & $\begin{array}{l}\text { CVTS3 } \\
(2005)\end{array}$ & $\begin{array}{l}\text { Qualifizierungs- } \\
\text { panel } \\
(2010)\end{array}$ & $\begin{array}{l}\text { CVTS3 } \\
(2005)\end{array}$ & $\begin{array}{l}\text { Qualifizierungs- } \\
\text { panel } \\
(2010)\end{array}$ & $\begin{array}{l}\text { CVTS3 } \\
(2005)\end{array}$ & $\begin{array}{l}\text { Qualifizierungs- } \\
\text { panel } \\
(2010)\end{array}$ & $\begin{array}{l}\text { CVTS3 } \\
\text { (2005) }\end{array}$ & $\begin{array}{l}\text { Qualifizierungs- } \\
\text { panel } \\
(2010)\end{array}$ \\
\hline Insgesamt (10 und mehr Beschäftigte) & 43 & 50 & 26 & 32 & 12 & 5 & 18 & 13 \\
\hline 10-49 Beschäftigte & 38 & 45 & 27 & 35 & 13 & 5 & 22 & 16 \\
\hline 50-249 Beschäftigte & 56 & 63 & 25 & 26 & 10 & 8 & 9 & 3 \\
\hline 250 und mehr Beschäftigte & 73 & 82 & 14 & 15 & 6 & 2 & 7 & $1 *$ \\
\hline 1-9 Beschäftigte & - & 11 & - & 41 & - & 6 & - & 43 \\
\hline
\end{tabular}

* Geringe Fallzahl.

Quelle: FDZ der statistischen Ämter des Bundes und der Länder, CVTS3-Erhebung 2005; Berechnungen der Autoren. BIBB-Betriebspanel zu Qualifizierung und Kompetenzentwicklung, Erhebungswelle 2011.

Unternehmen, die keinen Bedarf an neuen Qualifikationen haben. ${ }^{8}$ Viele Unternehmen in Deutschland sind jedoch in der Aus- und/oder Weiterbildung aktiv. So haben im Jahr 2005 die weitaus meisten Unternehmen in Deutschland mit zehn und mehr Beschäftigten betriebliche Bildungsmaßnahmen durchgeführt (Tabelle 1). Dabei boten $43 \%$ der Unternehmen sowohl Aus- als auch Weiterbildungsmaßnahmen ${ }^{\circledR}$ an und verfolgten damit eine komplementäre Strategie. 38 \% spezialisierten sich auf Aus- oder Weiterbildung. Eine Minderheit von $18 \%$ war nicht aktiv. Erwartungsgemäß (und durch andere Untersuchungen belegt, vgl. zum Beispiel Bellmann et al. 2010) wurde eine komplementäre Strategie in Großbetrieben sehr viel häufiger als in kleineren Unternehmen verfolgt. Die Ausbildung hat meist positive Auswirkungen auf die Weiterbildung in einem Unternehmen, wie multivariate Analysen mit unterschiedlichen Datensätzen zeigen. So steigt die Wahrscheinlichkeit, Weiterbildung anzubieten, mit dem Auszubildendenanteil an der Belegschaft (vgl. zum Beispiel Anger/Werner 2009; Bellmann et al. 2001; Kuckulenz/Meyer 2006; Leber 2002). Dies könnte ein Hinweis darauf sein, dass in der Ausbildung besonders engagierte Unternehmen über interne Kapazitäten und Organisationsstrukturen verfügen, die sie auch für die Weiterbildung und die Einarbeitung neuer Beschäftigter nutzen und umgekehrt. Der Auszubildendenanteil wäre somit ein Prädiktor für eine Lernkultur im Unternehmen, die sowohl Aus- als auch Weiterbildung umfasst (Anger/Werner 2009).

Neuere Zahlen des BIBB-Betriebspanels zu Qualifizierung und Kompetenzentwicklung ${ }^{\circledR}$ für 2010 bestätigen die in CVTS3 ermittelte Struktur der Aus- und Weiterbildung. ${ }^{(1)}$ Zusätzlich füllen sie die Lücke für Unternehmen mit weniger als zehn Beschäftigten: Diese sind nur wenig bildungsaktiv und konzentrieren sich meist auf Weiterbildung. Nur etwas mehr als jedes zehnte Kleinstunternehmen bietet Ausund Weiterbildung an. Deutlich wird auch ihre insgesamt geringe Beteiligung an der Ausbildung.

\subsection{Nutzung verschiedener Lernformen}

Berufliche Bildung kann in verschiedenen Lernformen stattfinden. Eine Vielfalt von Lernformen im Unternehmen kann auf eine intensive Auseinandersetzung mit dem Thema Bildung hindeuten. Sie ermöglicht darüber hinaus, für unterschiedliche Bedürfnisse und Beschäftigtengruppen jeweils besonders geeignete Maßnahmen anzubieten (Grünert et al. 2011; Moraal et al. 2009). In CVTS3 wurden

(8) So gaben in CVTS3 vier Fünftel der weiterbildungsabstinenten Unternehmen an, dass die Fähigkeiten ihrer Beschäftigten ausreichend waren und somit kein Weiterbildungsbedarf bestand (Cedefop 2010).

(9) Weiterbildende Unternehmen haben 2005 mindestens eine der nachfolgend aufgeführten Weiterbildungsaktivitäten durchgeführt: interne Kurse; externe Kurse; Weiterbildung am Arbeitsplatz; Weiterbildung durch Job-Rotation und Austauschprogramme; Teilnahme an Lern- und Qualitätszirkeln; selbstgesteuertes Lernen; Besuch von Informationsveranstaltungen. Ausbildende Unternehmen haben mindestens einen Auszubildenden im Jahresverlauf beschäftigt.

(10) Das BIBB-Qualifizierungspanel (Gerhards et al. 2012) ist eine repräsentative jährliche Befragung von 2.000 Betrieben aller Betriebsgrößenklassen und Wirtschaftszweige in Deutschland, die erstmalig 2011 durchgeführt wurde. Die Unternehmen werden zu ihren betrieblichen Aus- und Weiterbildungsaktivitäten und der Personalrekrutierung befragt. Wir bedanken uns bei der Projektgruppe für die Auswertungen.

(11) Abweichungen ergeben sich vor allem aus der unterschiedlichen Grundgesamtheit (CVTS3: Unternehmen mit zehn und mehr Beschäftigten in den Wirtschaftszweigen C-K und O (NACE Rev. 1.1); BIBB-Qualifizierungspanel: alle Betriebe mit mindestens einem sozialversicherungspflichtig Beschäftigten) und Anpassungen bei der Gewichtung an unterschiedliche Größenklassen und Branchen. 


\section{Nutzung verschiedener Instrumente der Weiterbildungsorganisation in Deutschland und in der EU 27 (2005)}

in Prozent der weiterbildenden Unternehmen

\begin{tabular}{|c|c|c|c|c|c|}
\hline & \multirow[t]{2}{*}{ EU 27} & \multicolumn{4}{|c|}{ Deutschland } \\
\hline & & Insgesamt & $\begin{array}{c}10-49 \\
\text { Beschäftigte }\end{array}$ & $\begin{array}{c}50-249 \\
\text { Beschäftigte }\end{array}$ & $\begin{array}{l}250 \text { und mehr } \\
\text { Beschäftigte }\end{array}$ \\
\hline $\begin{array}{l}\text { Für Weiterbildung verantwortliche Person / } \\
\text { Organisationseinheit im Unternehmen* }\end{array}$ & 42 & 36 & 32 & 42 & 66 \\
\hline Weiterbildungsbudget & 32 & 26 & 19 & 38 & 60 \\
\hline Weiterbildungsplan & 34 & 22 & 16 & 29 & 56 \\
\hline Formalisierte Mitarbeitergespräche* & 30 & 19 & 14 & 24 & 47 \\
\hline Systematische Analysen zum Qualifikationsbedarf* & 26 & 16 & 13 & 18 & 42 \\
\hline Nutzung von externen Beratungsleistungen zur Weiterbildung* & 19 & 8 & 8 & 7 & 13 \\
\hline Bildungszentrum & 15 & 7 & 5 & 8 & 21 \\
\hline \multicolumn{6}{|l|}{ Evaluationsinstrumente: } \\
\hline Messung der Zufriedenheit* & 37 & 21 & 18 & 25 & 50 \\
\hline Bewertung der Leistung derTeilnehmenden* & 37 & 16 & 15 & 14 & 28 \\
\hline Test derTeilnehmenden* & 32 & 13 & 13 & 15 & 11 \\
\hline Messung der Auswirkungen* & 21 & 11 & 11 & 11 & 13 \\
\hline
\end{tabular}

* Die angegebenen Prozentangaben beziehen sich auf die Summe der Antworten zu den Antwortoptionen „regelmäßig“ und "oft" bzw. bei den Evaluationsinstrumenten "nach jeder Maßnahme" und "nach den meisten Maßnahmen“"

Quelle: Eurostat, CVTS3 (Abrufdatum: 6.2.2012)

acht Lernformen unterschieden (Ausbildung und sieben Weiterbildungsformen; siehe Fußnote 9). ${ }^{\mathbb{B}}$ Im Durchschnitt aller Unternehmen wurden fast drei verschiedene Lernformen genutzt. Allerdings boten etwas mehr als ein Drittel der Unternehmen gar keine oder höchstens eine Lernform an (Abbildung 1). Insbesondere in kleinen Un ternehmen war die Anzahl an Lernformen gering. Wurde nur eine Form angeboten, war dies in $71 \%$ der Unternehmen die Erstausbildung, mit deutlichem Abstand folgten Informationsveranstaltungen mit einem Anteil von $14 \%$. In $28 \%$ der Unternehmen - darunter viele Großunternehmen - herrschte hingegen eine große Vielfalt an Lernformen. Unternehmen, die sowohl aus- als auch weiterbildeten, boten besonders viele verschiedene Formen an, $51 \%$ nutzten fünf und mehr Formen. Bei Unternehmen, die nur weiterbildeten, war der entsprechende Anteil mit $22 \%$ sehr viel niedriger.

\subsection{Organisation der Bildung}

Aus- und Weiterbildung kann in Unternehmen durch eine Reihe von Instrumenten institutionalisiert beziehungsweise formalisiert werden. In CVTS3 wurden insgesamt elf Fragen (Statistische Ämter des Bundes und der Länder 2006 Fragen $4.1-4.11$, S. 12f.) an weiterbildende Unternehmen zu verschiedenen Elementen der Weiterbildungsorganisation gestellt. Die Nutzung solcher Instrumente weist auf eine gewisse Professionalisierung der Bildungsmaßnahmen
ABB. 1

\section{Anzahl der verschiedenen Lernformen in Unternehmen 2005}

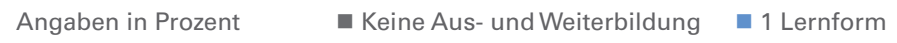

a 2 bis 4 Lernformen 5 und mehr Lernformen

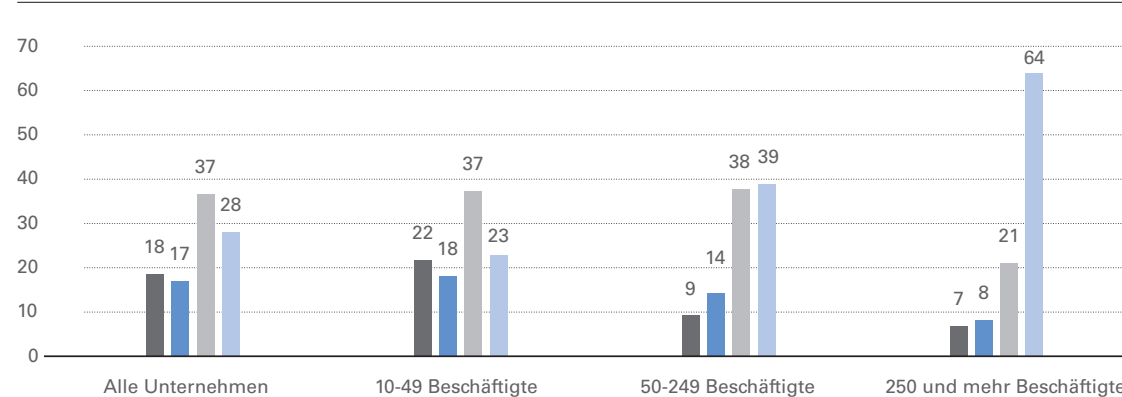

Quelle: FDZ der statistischen Ämter des Bundes und der Länder, CVTS3-Erhebung 2005; Berechnungen der Autoren.
(22) Angebot der verschiedenen Lernformen in allen Unternehmen: Informationsveranstaltungen $58 \%$, Ausbildung $55 \%$, externe Kurse $49 \%$, Weiterbildung am Arbeitsplatz $48 \%$, interne Kurse $39 \%$, Lern- und Qualitätszirkel $16 \%$, selbstgesteuertes Lernen $15 \%$, Job-Rotation und Austauschprogramme $9 \%$. 
ABB. 2

\section{Anzahl der verschiedenen Instrumente* der Weiterbildungsorganisation in weiterbildenden Unternehmen 2005 \\ Angaben in Prozent \\ - Keine WB-Instrumente $\quad 1$ WB-Instrument \\ - 2 bis 4 WB-Instrumente 5 und mehrWB-Instrumente}

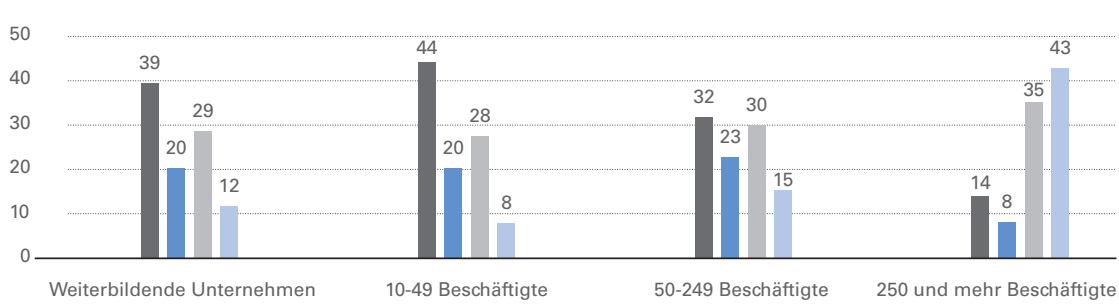

*Die erfragten Instrumente waren Weiterbildungsbudget, Weiterbildungsplan, formalisierte Mitarbeitergespräche, systematische Analysen zum Qualifikationsbedarf, externe Beratungsleistungen zur Weiterbildung, Bildungszentrum. Die vier Evaluationsinstrumente Messung der Zufriedenheit, Bewertung der Leistung der Teilnehmenden, Test derTeilnehmenden, Messung der Auswirkungen wurden zusammengefasst, sodass es höchstens acht verschiedene Instrumente in einem Unternehmen geben kann.

Quelle: FDZ der statistischen Ämter des Bundes und der Länder, CVTS3-Erhebung 2005: Berechnungen der Autoren. es sich dabei um eine Person oder Organisationseinheit, die für betriebliche Weiterbildung verantwortlich ist, in $24 \% \mathrm{der}$ Unternehmen wird zumindest eine Evaluationsform ${ }^{\circledR}$ eingesetzt, $21 \%$ dieser Unternehmen haben ein Jahresbudget für die Aus- und Weiterbildung, das Mittel für die betriebliche Weiterbildung mit einschloss. Auffällig ist, dass in Unternehmen, die aus- und weiterbilden, der Anteil der Unternehmen mit Personen, die für betriebliche Weiterbildung verantwortlich sind, sowie mit Jahresbudgets und Weiterbildungsplänen deutlich höher ist als in Unternehmen, die nur weiterbilden. Dies könnte ein Hinweis darauf sein, dass ihr Vorhandensein insbesondere der Ausbildung geschuldet ist.

\subsection{Einfluss der Betriebsräte auf Bildungs- maßnahmen}

Betriebsräte haben durch entsprechende gesetzliche Regelungen ( $\$ \$ 96$ bis 98 des Betriebsverfassungsgesetzes) die Möglichkeit, über betriebliche Bildungsmaßnahmen mitzubestimmen, zum Beispiel über die Inhalte und Zielgruppen der Weiterbildung. ${ }^{-1} 17 \%$ der weiterbildenden Unternehmen hatten 2005 einen Betriebsrat. Es zeigt sich jedoch nur ein geringes Engagement für berufliche Bildungsfragen. 65 \% der weiterbildenden Unternehmen mit einem Betriebsrat beschäftigten sich nicht mit den in CVTS3 abgefragten Elementen zum Weiterbildungsmanagement. Bei den restlichen Unternehmen nahm der Betriebsrat insbesondere Einfluss auf die Auswahl der Zielgruppen, die an Weiterbildung teilnehmen sollen, auf die Inhalte und Themen der Weiterbildung sowie die Ziele und Prioritäten. Dennoch lassen sich deutliche Hinweise für einen positiven Einfluss der Existenz eines Betriebsrats finden. So weisen Unternehmen mit einem Betriebsrat zum Beispiel höhere Nutzungsgrade bei der Vielfalt der Lernformen und bei der Zahl der genutzten Instrumente der Weiterbildungsorganisation auf. ${ }^{\circledast}$ 27-Durchschnittswerten (Tabelle 2). Dies deutet auf eine geringe Institutionalisierung der Weiterbildungsorganisation in Unternehmen in Deutschland hin. Die Unternehmensgröße hat hier einen großen Einfluss - kleinere Unternehmen, die meist seltener und unregelmäßiger Bildungsmaßnahmen anbieten, nutzen solche Elemente weniger als große Unternehmen.

Fragt man nach der Intensität der Nutzung, fällt zunächst auf, dass ein großer Teil der weiterbildenden Unternehmen überhaupt keine Maßnahmen ergreift, um Weiterbildung organisatorisch im Unternehmen zu verankern (Abbildung 2). In diesen Unternehmen scheint sich bei einem Weiterbildungsbedarf eher ad-hoc um das passende Angebot gekümmert zu werden. Dies mag auch damit zusammenhängen, dass Weiterbildung ja kein primäres Unternehmensziel ist, sondern eher eine unterstützende Funktion hat, die bei Bedarf eingesetzt wird, um zum Beispiel auf technologische oder strukturelle Veränderungen oder neue Qualifikationsbedürfnisse zu reagieren. Hohe Institutionalisierungsgrade gibt es nur in Großunternehmen. Deutlich mehr als die Hälfte der kleinen und mittleren Unternehmen nutzt höchstens ein Instrument. In rund einem Drittel dieser Unternehmen handelt
(13 Allerdings handelt es sich dabei überwiegend um eine einfache Messung der Zufriedenheit der Beschäftigten mit der Weiterbildungsmaßnahme. Diese Art der Evaluation ist wenig aussagekräftig und relativ anspruchslos.

(44) Allerdings wird oft beklagt, dass Betriebsräte sich nur selten auf dem Gebiet der betrieblichen Aus- und Weiterbildung engagieren. Da die Regulierungsdichte und Vorgaben bei der Ausbildung sehr viel größer als bei der Weiterbilten und Aufgaben von Betriebsräten in beiden Bereichen. Siehe hierzu das BIBB-Forschungsprojekt „Betriebliche Aus- und Weiterbildungsaktivitäten als Handlungsfeld von Arbeitnehmervertretungen - Selbstverständnis, Handlungsstrategien, Wirkungen“ (URL: http://www.bibb.de/de/ wlk57131.htm [letzter Zugriff: 27.4.2012]).

(15) Siehe hierzu auch den Beitrag von Berger in diesem Heft. Er identifiziert ebenfalls betriebliche Weiterbildung als ein nachrangiges Handlungsfeld von Betriebsräten, dennoch haben Betriebe mit einem Betriebsrat insbesondere im Bereich der Weiterbildung intensivere Bildungsaktivitäten als betriebsratslose Betriebe. dung sind, unterscheiden sich auch die Einflussmöglichkei- 


\subsection{Verzahnung von Aus- und Weiterbildung in Unternehmen}

Die Ausbildung in einem Unternehmen hat positive Auswirkungen auf die Weiterbildung. So zeigen sich sowohl bei den Teilnahmequoten an Weiterbildungskursen und anderen Formen als auch bei der Stundenzahl in Kursen je Beschäftigten beziehungsweise je Teilnehmenden bei den ausund weiterbildenden Unternehmen höhere Werte im Vergleich zu Unternehmen, die nur weiterbilden und keine Auszubildenden haben.

Dies führt zu der Frage, inwieweit es in den Unternehmen zu einer Zusammenarbeit in der Aus- und Weiterbildung kommt. Die Forderung nach einer besseren Verzahnung von Aus- und Weiterbildung, umso mehr Durchlässigkeit im Bildungssystem zu ermöglichen, wird von politischer Seite immer wieder aufgestellt. Meistens wird hier zwar an den Übergang zwischen beruflicher Bildung und Hochschulbildung gedacht, aber auch auf betrieblicher beziehungsweise beruflicher Ebene sind Verbindungen möglich. Zu denken wäre zum Beispiel an eine bessere Verknüpfung von Ausbildung und Aufstiegsfortbildung und verbesserte Optionen zur Anrechnung bereits erworbener Qualifikationen, um Lernbrüchen entgegenzuwirken und das lebenslange Lernen zu fördern. Die Bundesregierung hat hier mit ihren Initiativen im Innovationskreis Weiterbildung Akzente gesetzt, z. B. durch die Förderung von Wahl- und Zusatzqualifikationen, ${ }^{\circ}$ die als wichtige Schnittstellen zwischen der beruflichen Erstausbildung und der Weiterbildung gesehen werden.

In der CVTS3-Zusatzerhebung wurden einige Fragen gestellt, um den Grad der Verzahnung von Aus- und Weiterbildung in Unternehmen zu ermitteln. Es wurden nur weiterbildende Unternehmen befragt, fast drei Viertel bildeten auch in einem oder mehreren Berufen aus. Vier Indikatoren beschreiben den Grad der Verzahnung (Angaben jeweils in Prozent der aus- und weiterbildenden Unternehmen):

- Lediglich in $25 \%$ der Unternehmen erfolgte die Planung und Organisation der Ausbildung in Zusammenarbeit und Absprache mit der Planung und Organisation der Weiterbildung.

- In immerhin $40 \%$ der Unternehmen gab es Ausbildungspersonal, das auch für die berufliche Weiterbildung eingesetzt wurde.

- In $25 \%$ der Unternehmen wurden Berufe ausgebildet, die Wahlqualifikationen beinhalten. In 68 \% dieser Unternehmen wurden die Inhalte der Wahlqualifikationen auch für Weiterbildungszwecke genutzt.

- Wesentlich mehr Unternehmen boten in der Ausbildung Zusatzqualifikationen (45 \%) an. $74 \%$ nutzten die Inhalte der Zusatzqualifikationen auch für Weiterbildungszwecke.

Das Ausmaß der Verzahnung von beruflicher Ausbildung mit betrieblicher Weiterbildung ist in den meisten Unternehmen nicht sehr hoch. In $34 \%$ der Unternehmen wurde keine der angesprochenen Maßnahmen genutzt, in $31 \%$ höchstens eine Maßnahme. Von einer engen Verzahnung ist in lediglich $11 \%$ der Unternehmen auszugehen, die entweder drei ( $9 \%)$ oder alle vier untersuchten Maßnahmen ( $2 \%)$ nutzten.

$\mathrm{Zu}$ einer besseren Durchlässigkeit im Bildungssystem kann auch die Beteiligung der Auszubildenden an den im Unternehmen angebotenen Weiterbildungsmaßnahmen beitragen. In den aus- und weiterbildenden Unternehmen, die an der CVTS3-Zusatzerhebung teilnahmen, stehen die Weiterbildungsangebote in der Regel auch den Auszubildenden offen: Sie haben dabei insbesondere die Möglichkeit zur Teilnahme an Kursen, an der Weiterbildung am Arbeitsplatz ${ }^{(1)}$ und zum Besuch von Informationsveranstaltungen zum Zwecke der Weiterbildung. Kaum von Bedeutung sind hingegen das selbstgesteuerte Lernen, die Teilnahme an Lern- und Qualitätszirkeln und Job-Rotation, Austauschprogramme und Studienbesuche.

Obwohl nur in wenigen der aus- und weiterbildenden Unternehmen eine enge Verzahnung zwischen beruflicher Ausbildung und betrieblicher Weiterbildung existierte, war den befragten Verantwortlichen für die berufliche Bildung in den Unternehmen bewusst, dass eine solche Verzahnung künftig wichtiger wird. So stimmten $87 \%$ der Aussage zu, dass lebenslanges Lernen auch bedeutet, dass in Unternehmen die Ausbildung und die berufliche Weiterbildung besser aufeinander abgestimmt werden sollen.

\section{Fazit}

Wie gezeigt, beteiligen sich zwar viele Unternehmen an der Aus- und/oder Weiterbildung. Meist beschränken sie sich aber auf ein oder höchstens zwei unterschiedliche Angebote, nur wenige Unternehmen nutzen eine größere Zahl verschiedener Bildungsformen. Auch ergreifen nur relativ wenige Unternehmen Maßnahmen, um die Weiterbildung organisatorisch stärker im Unternehmen zu verankern, um sie so auf eine feste Grundlage zu stellen und bei Bedarf schnell auf Qualifikationsbedürfnisse reagieren zu können. Aus- und Weiterbildung stehen in vielen Unternehmen noch unverbunden nebeneinander. Wissen und Er-

(16 Einige Ausbildungsberufe beinhalten Pflicht- und Wahlqualifikationseinheiten. Die Pflichtqualifikationen müssen vermittelt werden; aus den Wahlqualifikationen kann ein Betrieb unter Berücksichtigung seiner betrieblichen Struktur auswählen. Zusatzqualifikationen richten sich an Auszubildende in einem staatlich anerkannten Ausbildungsberuf, finden während der Ausbildung statt und können zertifiziert werden. Sie gehen über die regulären Inhalte der Berufsausbildung (laut Ausbildungsordnung) hinaus.

(1) Diese ist aber zugleich ein wichtigerTeil ihrer Ausbildung, sodass nicht ganz klar ist, ob die befragten Unternehmen hier wirklich nur an Weiterbildung gedacht haben. 
fahrungen, die in einem Bereich gewonnen wurden, werden nicht gewinnbringend für den jeweils anderen Bereich genutzt. Größere Unternehmen schneiden in allen angesprochenen Kategorien erheblich besser ab als kleinere Unternehmen. Die Ausbildung hat einen positiven Einfluss auf das Weiterbildungsangebot.

Das deutsche Bildungssystem ist weitgehend segmentiert. Im dualen Ausbildungssystem zeigen sich die negativen Auswirkungen der zunehmenden Segmentierung, insbesondere durch den Rückzug vor allem der kleineren Unternehmen von der Ausbildung und der Ausgrenzung eines erheblichen Teils der Jugendlichen, die in das Übergangssystem verwiesen werden. Einige europäische Länder verfolgen hier eine andere Strategie, um die meisten Schulabgänger ohne Umwege direkt in eine berufliche Ausbildung zu leiten. In den Niederlanden nimmt das duale Ausbildungsprinzip eine große Rolle ein. Schulische und betriebliche Ausbildungspfade ${ }^{\circledR}$ sind dort in einem harmonisierten Gesamtsystem integriert, das Flexibilität beim Wechsel der Ausbildungspfade ermöglicht. Das System bietet durch einen schwellenfreien Zugang zumindest zu Niveau 1- oder 2-Ausbildungen allen Jugendlichen die Chance auf den Erwerb einer Qualifikation. Besondere Maßnahmen wie sie das deutsche Übergangssystem kennzeichnen, werden dadurch überflüssig. Die Durchlässigkeit zwischen beruflicher Aus- und Weiterbildung ist durch die Gliederung der Bildungsgänge in vier Qualifikationsstufen (Hilfskraft, angehende Fachkraft, Fachkraft, Fachkraft der mittleren Führungsebene) impliziert. Grundsätzlich kann jede Stufe über beide Zweige erreicht werden (Hoppe 2005). In der Schweiz ist besonders die Möglichkeit des Erwerbs der Berufsmaturität während der dualen Ausbildung interessant, die die Durchlässigkeit nach oben zur Fachhochschule ermöglicht und von vielen Jugendlichen genutzt wird (Ebner 2009).

In Deutschland bestehen erhebliche Ungleichheiten beim Zugang zu Aus- und Weiterbildung für Arbeitskräfte, je nachdem in welchem Betrieb sie arbeiten. Erschwerend kommt hinzu, dass sich Bildungsmaßnahmen in Unternehmen oftmals an deren Qualifikationsinteressen orientieren, ohne die gesamtwirtschaftlichen Erfordernisse und die individuellen Bedürfnisse der Beschäftigten nach grundlegenden, allgemein anwendbaren und auf andere Unternehmen übertragbaren Fähigkeiten ausreichend zu berücksichtigen. Hier wäre es wichtig, unternehmensunabhängige Bildungsangebote auch für solche Erwerbstätigengruppen zu schaffen, denen die betrieblichen Angebote nicht offenstehen. Das segmentierte Bildungssystem, in dem die verschiedenen Akteure vorrangig auf ihre eigenen Interessen bedacht sind, steht solchen Angeboten allerdings entgegen. Angesichts der Dynamik der Veränderungsprozesse in der globalisierten Welt sollte die berufliche Bildung in Deutschland einen anderen Stellenwert bekommen und das lebenslange Lernen stärker institutionalisiert werden, zum Beispiel durch die Nutzung von Kooperationen wie regionalen Qualifikationsnetzwerken oder einer Stärkung der tariflichen Weiterbildungspolitik.
Dies würde für die segmentierte Weiterbildungslandschaft bedeuten, dass ein Strategiewechsel von punktuellen ad-hocMaßnahmen hin zur Implementierung einer Infrastruktur für lebenslanges Lernen vollzogen wird, in die Unternehmen und Beschäftigte ebenso einbezogen sein müssen wie Bildungsanbieter und die öffentliche Hand.

(8) Ein Minimum an berufspraktischem Lernen ist in beiden Formen garantiert: In der betrieblichen Form werden mindestens $60 \%$ der Ausbildung in einem Unternehmen und der übrige Teil wird in der Schule absolviert. Die vollzeitschulische Ausbildung ist stärker theoretisch angelegt, der Anteil der berufspraktischen Ausbildung liegt zwischen 20 und $60 \%$ (Hoppe 2005). 


\section{LITERATUR}

Anger, C./Werner, D. (2009): Ergebnisse von CVTS und IW-Weiterbildungserhebung für Deutschland im Vergleich, in: Zeitschrift für Berufs- und Wirtschaftspädagogik 105, Beiheft 22, S. 125-148

Baethge, M. (2007): Stichwort "Übergänge“, in: DIE Zeitschrift für Erwachsenenbildung 14 (1), S. 24/25

Behringer, F./Käpplinger, B. (2011): Arbeitsplatznahe Lernformen und Lernortvielfalt in der betrieblichen Weiterbildung. Wachsende Bedeutung in ganz Europa oder deutsches Spezifikum?, in: Berufsbildung in Wissenschaft und Praxis 40 (1), S. 15-19

Behringer, F./Schönfeld, G. (2010): Betriebliche Weiterbildung in Deutschland Ein Vergleich mit den EU-Mitgliedstaaten auf der Grundlage der vier Kernindikatoren aus CVTS3, Bonn, http://www.bibb.de/dokumente/pdf/Fachbeitrag behringer-schoenfeld2010.pdf (Stand: 4.2.2012)

Beicht, U. (2010): Bedeutung und Wirksamkeit von Bildungsgängen des Übergangssystems, in: Bundesinstitut für Berufsbildung (BIBB) (Hrsg.): Datenreport zum Berufsbildungsbericht 2010. Informationen und Analysen zur Entwicklung der beruflichen Bildung, Bielefeld, S. 90-96

Bellmann, L./Düll, H./Leber, U. (2001): Zur Entwicklung der betrieblichen Weiterbildungsaktivitäten. Eine empirische Untersuchung auf der Basis des IABBetriebspanels, in: Reinberg, A. (Hrsg.): Arbeitsmarktrelevante Aspekte der Bildungspolitik, Nürnberg, S. 97-123

Bellmann, L./Krekel, E./Stegmaier, J. (2010): Aus- und Weiterbildung - Komplemente oder Substitute? Zur Bildungsbeteiligung kleinerer und mittlerer Betriebe in Deutschland, in: REPORT - Zeitschrift für Weiterbildungsforschung 33 (1), S. 41-54

Bundesinstitut für Berufsbildung (BIBB) (Hrsg.) (2012): Datenreport zum Berufsbildungsbericht 2012. Informationen und Analysen zur Entwicklung der beruflichen Bildung, Bielefeld

Bundesministerium für Bildung und Forschung (BMBF) (Hrsg.) (2012): Berufsbildungsbericht 2012, Bonn/Berlin

Centre européen pour le développement de la formation professionnelle (Cedefop) (Hrsg.) (2010): Employer-provided vocational training in Europe. Evaluation and interpretation of the third European Continuing Vocational Training Survey, Luxemburg, http://www.cedefop.europa.eu/EN/Files/5502 en.pdf (letzter Zugriff: 02.01.2012)

Ebner, C. (2009): Neue Wege für die duale Berufsausbildung - ein Blick auf Österreich, die Schweiz und Dänemark, in: WZB-Brief Arbeit (4), Berlin, http:// bibliothek.wzb.eu/wzbrief-arbeit/WZBriefArbeit042009_ebner.pdf (letzter Zugriff: 02.04.2012)

Gerhards, C./Mohr, S./Troltsch, K. (2012): Erhöht der Fachkräftemangel die Weiterbildungsbeteiligung von Betrieben? Analysen auf der Basis des BIBBQualifizierungspanels, in: Berufsbildung in Wissenschaft und Praxis 41 (1), S. $19-22$

Grünert, H./Wiener, B./Winge, S. (2011): Zusammenarbeit von Betrieben und Bildungsträgern in der beruflichen Weiterbildung, Halle (Saale)

Hoppe, M. (2005): Merkmale und Besonderheiten vollzeitschulischer Berufsausbildung in den Niederlanden, Österreich und Dänemark, in: Berufsbildung in Wissenschaft und Praxis 34 (4), S. $51-54$

Konsortium Bildungsberichterstattung (Hrsg.) (2006): Bildung in Deutschland Ein indikatorengestützter Bericht mit einer Analyse zu Bildung und Migration, Bielefeld

Kuckulenz, A./Meyer, J. (2006): Die Entscheidung über betriebliche Weiterbildungsinvestitionen. Eine empirische Analyse mit dem Mannheimer Innovationspanel, in: ZEW Discussion paper (06-089), Mannheim, http://doku.iab.de/ externe/2007/k070813n17.pdf (letzter Zugriff: 18.01.2010)
Leber, U. (2002): Betriebliche Weiterbildung von Männern und Frauen, in: Engelbrech, G. (Hrsg.): Arbeitsmarktchancen für Frauen. Beiträge zur Arbeitsmarkt- und Berufsforschung (258), Nürnberg, S. 175-191

Moraal, D./Lorig, B./Schreiber, D./Azeez, U. (2009): Ein Blick hinter die Kulissen der betrieblichen Weiterbildung in Deutschland. Daten und Fakten der nationalen CVTS3-Zusatzerhebung. BIBB Report 7, http://www.bibb.de/ dokumente/pdf/a12_bibbreport_2009_07.pdf (letzter Zugriff: 26.01.2012)

Sauter, E. (2003): Übergänge zwischen Ausbildung und Weiterbildung. Ordnungspolitische Rahmenbedingungen und betriebliche Strategien, in: Ausbilder-Handbuch 64. Erg-Lfg., Oktober, S. 1-20

Seyda, S./Werner, D. (2012): IW-Weiterbildungserhebung 2011 - Gestiegenes Weiterbildungsvolumen bei konstanten Kosten, in: IWTrends 39 (1), S. 1-19 Statistische Ämter des Bundes und der Länder (2006): Berufliche Weiterbildung in Unternehmen 2005. Fragebogen, Wiesbaden, http://www. forschungsdatenzentrum.de/bestand/cvts/cf/2006/fdz_cvts_cf_2006_ fragebogen.pdf (letzter Zugriff: 21.12.2011)

Stegmaier, J. (2010): Betriebliche Berufsausbildung und Weiterbildung in Deutschland, Nürnberg, http://datenreport.bibb.de/IAB-Expertise_fuer_den_ Datenreport_zum_Berufsbildungsbericht_2011.pdf (letzter Zugriff: 18.06.2010) Thelen, K./Busemeyer, M. R. (2012): Institutional Change in German Vocational Training: From Collectivism toward Segmentalism, in: Busemeyer, M. R./Trampusch, C. (Hrsg.): The Political Economy of Collective Skill Formation, New York, S. 68-100

Trampusch, C. (2010): Employers, the state and the politics of institutional change: Vocational education and training in Austria, Germany and Switzerland, in: European Journal of Political Research 49 (4), S. 545-573

Troltsch, K. (2010): Verlauf und Nachhaltigkeit betrieblicher Ausbildungsbeteiligungen zwischen 1999 und 2008, in: Bundesinstitut für Berufsbildung (BIBB) (Hrsg.): Datenreport zum Berufsbildungsbericht 2010. Informationen und AnaIysen zur Entwicklung der beruflichen Bildung, Bielefeld, S. 195-200

Ulrich, J./Flemming, S./Granath, R.-O./Krekel, E. M. (2011): Verbesserte Ausbildungschancen für Jugendliche, zunehmende Rekrutierungsprobleme für Betriebe. Die Entwicklung des Ausbildungsmarktes im Jahr 2011, Bonn, http:// www.bibb.de/dokumente/pdf/internetbeitrag_ausbildungsmarkt_2011_15_12_2011.pdf (letzter Zugriff: 27.03.2012)

\section{AUTOREN}

DICK MORAAL, Dipl. Pol., ist wissenschaftlicher Mitarbeiter im Bundesinstitut für Berufsbildung (BIBB), Arbeitsbereich „Kosten, Nutzen, Finanzierung“. Arbeitsschwerpunkte: Betriebliche Weiterbildung im internationalen Vergleich, Kosten der beruflichen Weiterbildung.

moraal@bibb.de

GUDRUN SCHÖNFELD, M. A., ist wissenschaftliche Mitarbeiterin im BIBB, Arbeitsbereich „Kosten, Nutzen, Finanzierung“. Arbeitsschwerpunkte: Betriebliche Weiterbildung im internationalen Vergleich, Kosten der betrieblichen Aus- und Weiterbildung.

schoenfeld@bibb.de 